\title{
Orientation Invariant Pattern Recognition by Pigeons (Columba livia) and Humans (Homo sapiens)
}

\author{
Juan D. Delius and Valerie D. Hollard \\ Universität Konstanz
}

\begin{abstract}
The orientation invariance of visual pattern recognition in pigeons and humans was studied using a conditioned matching-to-sample procedure. A rotation effect, a lengthening of choice latencies with increasing angular disparities between sample and comparison stimuli, was replicated with humans. The choice speed and accuracy of pigeons was not affected by orientation disparities. Novel mirror-image stimuli, rotation of sample shapes, a delayed display of comparison shapes, and a mixed use of original and reflected sample shapes did not lead to a rotation effect in pigeons. With arbitrarily different odd comparison shapes, neither humans nor pigeons showed a rotation effect. Final experiments supported the possibility that the complete absence of a rotation effect in pigeons is because they are relatively better than humans at discriminating mirror-image shapes compared with arbitrary shapes.
\end{abstract}

The term orientation invariance refers to the process whereby stimulus shapes can be recognized visually or haptically as being identical or being different even when their orientations in space differ. It is a competence assumed to be in frequent demand among many vertebrate animals living under natural conditions. Delius (unpublished observation, 1969), for example, was impressed by how quickly and accurately wild-living spider monkeys recognized a large number of conspecific individuals regardless of the highly variable spatial attitudes that both observer and partners could assume. Nevertheless, not much is known about the occurrence or the quality of this capacity in species other than humans. Under certain circumstances, visual rotation invariance in humans is associated with an effect known as mental rotation. Mental rotation is said to occur when discrimination of shapes presented with orientation disparities yields a performance decrement (more choice errors, longer choice latencies, or both). The effect has attracted much investigation over the past decades (Cooper, 1975; Corballis, 1988; Shepard \& Cooper, 1982; Shepard \& Metzler, 1971). The persisting interest has been partly fu-

Juan D. Delius and Valerie D. Hollard, Allgemeine Psychologie, Universität Konstanz.

Valerie D. Hollard is now at the Native Forest Restoration Trust, Green Bay, Auckland, New Zealand.

This research was supported by grants from the Deutsche Forschungsgeneinschaft. Valerie D. Hollard held visiting scholarships from Heinrich Hertz Stiftung and the Deutsche Akademische Austauschdienst. We thank Christoph Werner, Anette Lohmann, Petra Ohman, and Monika Remy (deceased) for running some of the experiments. We also are grateful to M. Siemann and J. M. Findlay for many helpful suggestions and G. Latini for language corrections. Juan D. Delius also thanks J. M. Findlay for sabbatical hospitality at the Department of Psychology, University of Durham, England, while the article was being prepared.

Correspondence concerning this article should be addressed to Juan D. Delius, Allgemeine Psychologie, Universität Konstanz, D-78434 Konstanz, Germany. eled by the fact that the mental rotation effect is viewed by some as a prototype cognitive phenomenon that is based on an analogical, or imagery-based, form of information processing (Kosslyn, Pinker, Smith, \& Schwartz, 1979), whereas others. vigorously dispute this (Pylyshyn, 1981). It is also true that the mental rotation performance of humans correlates with their intelligence quotient as measured by standard tests (Petrusic, Varro, \& Jamieson, 1978; Pellegrino \& Kail, 1982). Moreover, an orientation invariance capability has been found to be difficult to implement in artificial vision systems (Gonzales \& Wintz, 1987; Messner \& Szu, 1985).

A first, albeit indirect, hint that pigeons might be special concerning orientation invariance competences arose in a study of their long-term memory. It incidentally showed that they had unexpected difficulties with learning to discriminate an oblique cross $\times$ from an upright cross + , a task that seemed trivially simple to the experimenters (Delius \& Emmerton, 1978). The suspicion that pigeons might be more inclined to disregard the orientation features of visual stimuli than humans was bolstered by the subsequent finding that pigeons, by contrast, had no difficulty in learning to discriminate an angle $<$ from a cross $\times$ (Delius, 1979; see also Lohmann, Delius, Hollard, \& Friesel, 1988). Later, we reported briefly some initial results suggesting that when discriminating misaligned mirror-image shapes in closely similar experiments, pigeons, differently from humans, did not evince a mental rotation effect (Hollard \& Delius, 1982). The finding was that pigeons were able to discriminate mirror-image shapes equally fast and well regardless of orientation disparities. Even highly intelligent humans could not match the birds' performance. Because the relevant experiments and their results are essential for the comprehension of those described here, they are briefly recapitulated (as parts of Experiments 1 and 2). This permitted us to include certain significant details that had to be omitted from the short original report. The main purpose of this article, however, is to present the results of a series of further experiments carried out with both pigeons and hu- 
mans. They were intended to elucidate more clearly how and why the two species differ in rotation invariance competences.

\section{Experiment 1: Mirror-Image and Arbitrary Odd Stimuli in Humans}

Many authors have described the mental rotation effect occurring when human participants were confronted with the discrimination of mirror-image shapes subject to orientation disparities (Corballis, 1988; Shepard \& Cooper, 1982). However, the mental rotation effect is not a universal finding in orientation invariance experiments. Several reports indicate that the mental rotation effect does not arise when arbitrarily different shapes, rather than mirror-image shapes, are used as odd comparison stimuli (Corballis \& Nagourney, 1978; Eley, 1983; Tilgner \& Hauske, 1980; White, 1980). With such stimuli the accuracy and speed of choices are nearly constant and independent of orientation disparities between standard and comparison stimuli. For valid comparisons, it was important to demonstrate that these two findings would replicate using the same methods and the same stimuli that we intended to use with pigeons in the subsequent experiments.

\section{Method}

Mirror-image odd stimuli. Twenty-two college and university students $(11$ women and 11 men, mean age $=21$ years $)$ participated. They either volunteered or were completing a course requirement. A random half of the participants were assigned to a matching-to-sample group; the other half were assigned to an oddity-from-sample group.

The apparatus used was a vertical panel $(45 \times 45 \mathrm{~cm})$ with a row of three translucent keys $(2.5 \mathrm{~cm}$ in diameter, centers $7 \mathrm{~cm}$ apart, $18 \mathrm{~cm}$ above the base; see Figure 1). Stimuli were backprojected onto the keys with an automatic slide projector. The stimulus shapes were drawn with black ink on white paper, and all had the same surface area. They were meant to be vaguely but not closely similar to letters or numerals in style (see Figure 1). Reduced photographic negatives of these shapes were mounted, three at a time, on special slide frames. Projected on the keys, the shapes appeared as white figures of about $1 \times 1 \mathrm{~cm}$ on a dark background surround. The stimulus display on each of the keys was controlled by solenoid shutters located directly behind them. Photocells within the slide projector detected the presence or absence of separate perforations in the slide frames coding the stimulus triplets they bore. A microcomputer (DEC LSI 11) received this information, as well as that signaling key activations. Through a specially made interface, it controlled the events within the experimental sessions and recorded the stimulus and response data on a trial-by-trial basis. The program was written in Focal supplemented with Assembler routines.

The participants sat comfortably in a chair with an adjustable headrest; the conditioning panel was about $50 \mathrm{~cm}$ away from their eyes. They used a hand-held, pencillike pointer to press the keys and were first given written instructions and 10 training trials. If needed, additional verbal explanations were provided. The participants were told that each correct response would be credited with one pfennig (equivalent to about half a cent).
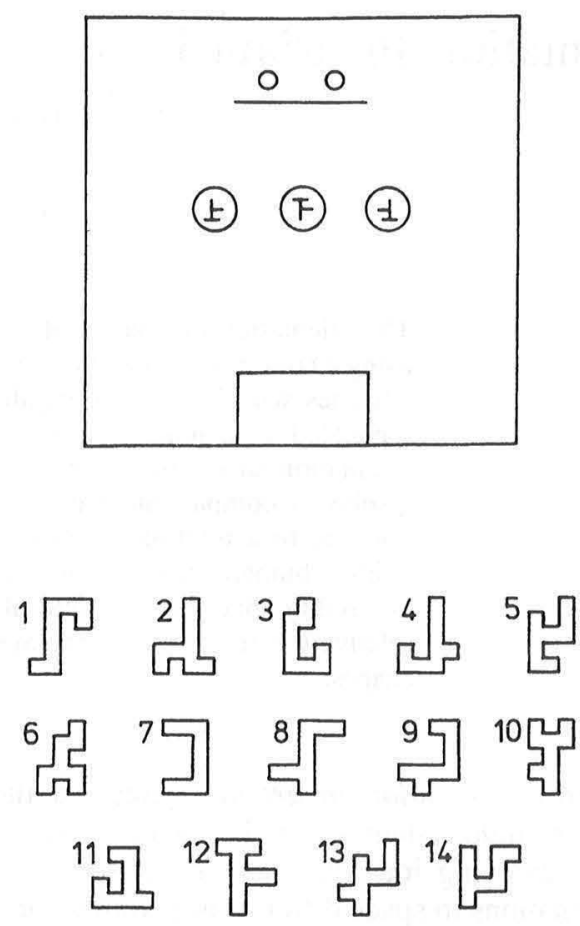

Figure 1. The conditioning panel (top) and the visual shapes (bottom) used in Experiment 1 with humans and in Experiments 2-4 with pigeons.

The stimulus triplets were assembled from the two shapes ( 8 and 11; see Figure 1) and their vertical axis reflections (mirror images). A sample shape was always displayed on the center key. In one half of the triplets, the matching comparison shape was displayed on the right-side key and in the other half on the left-side key, with the odd mirror-image comparison shape being accordingly displayed on the alternative side key. The sample shapes were shown in the standard $0^{\circ}$ orientation depicted in Figure 1 . The respective comparison shapes were both shown rotated on the projection plane by an angle relative to the sample shape orientation. This angular disparity was either $0^{\circ}, 45^{\circ}, 90^{\circ}, 135^{\circ}$, or $180^{\circ}$ clockwise in different triplets (see Figure 2C). Altogether, there were 20 different stimulus triplets. Of each triplet there were two replicate slides. Within each session the 40 slides were shown in randomized sequences with the constraint that odd-right and odd-left triplets followed each other quasi-randomly (Gellermann, 1933). Two sessions separated by a brief rest period were administered to each participant.

A trial began with the display of a sample shape on the center key. When the participant had pressed this key five times, the two side shutters opened and two comparison shapes appeared on the side keys. Matching-to-sample group participants were instructed to choose the key displaying the identical form; oddity-fromsample group participants were required to choose the key displaying the reflected shape. A single side-key response made all the shapes disappear. A correct choice lit one of the two bulbs located above the keys for $3 \mathrm{~s}$ and operated noisily an empty solenoid feeder (explained later in Experiment 2). An incorrect side-key choice led to darkness as the second light bulb extinguished for $3 \mathrm{~s}$. The time that elapsed between the onset of the comparison stimuli and the response to one of them was recorded as reaction latency. A 5-s interval preceded the next trial that began again with the presentation of a sample stimulus. 


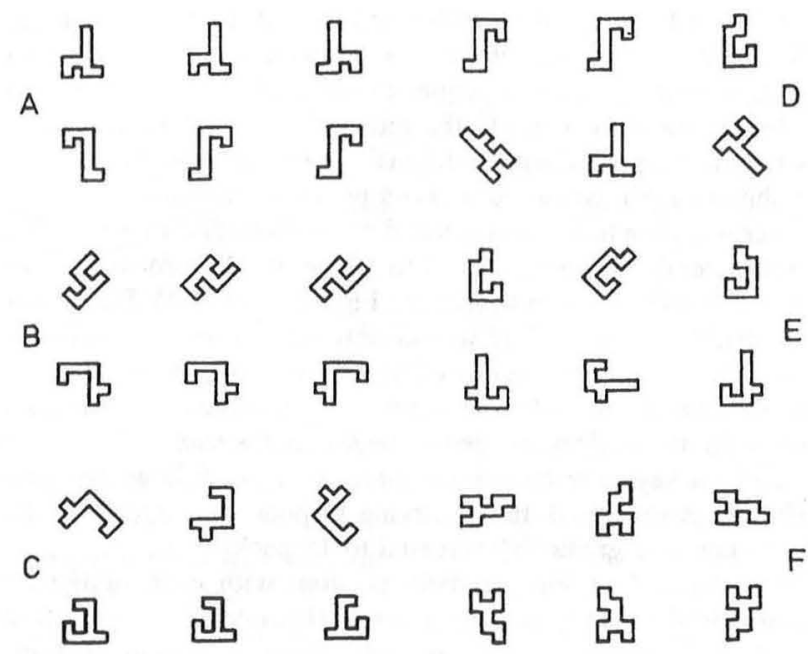

Figure 2. Examples of stimulus triplets used in Experiment 1 (C bottom, D) with humans and in Experiment 2 (A, B, C), Experiment $3(\mathrm{D}, \mathrm{E})$, and Experiment $4(\mathrm{D}, \mathrm{F})$ in pigeons.

For evaluation, the percentage errors and the mean reaction time corresponding to each type of triplet and for each participant was averaged across the repeated presentations of a given type of triplet. These mean error rates and reaction times were subjected to either analyses of variance (ANOVAs) or nonparametric tests.

Arbitrary odd stimuli. Twenty students (10 women and 10 men, average age $=23$ years) completing a course requirement served as participants; half of them were assigned to a matching group and the other half to an oddity group. The apparatus and procedures used were the same as described earlier except for the set of stimulus triplets used. Shapes 1 and 2 served as sample stimuli and matching comparisons. Shapes 3, 4, 5, 6, and 10 (see Figure 1) served as odd comparison stimuli according to a balanced randomized design (see Figure 2D). Five different odd shapes were used in an attempt to make the task approximately as difficult as the one involving mirror-image stimuli. Preliminary trials had shown that with only two such shapes, the task was trivially easy. The comparison shapes were again presented with one of the standard five orientation disparities $\left(0^{\circ}, 45^{\circ}, 90^{\circ}, 135^{\circ}\right.$, and $\left.180^{\circ}\right)$. Each of the 20 resulting triplets was shown four times to each participant in the course of two 40-trial sessions. The data were evaluated as described earlier.

\section{Results and Discussion}

Mirror-image odd stimuli. The results corresponding to Shapes 8 and 11 did not differ significantly. The results are thus shown pooled in Figure 3. As we expected, the mean reaction times lengthened as the orientation disparities of the comparison stimuli increased. This mental rotation effect was highly significant, $F(4,84)=22.76, p<.001$. The error rates revealed a slight but not significant concordant trend, which was not surprising because both instructions and pay-offs were aimed at ensuring a trade-off favoring accuracy rather than decision speed. Since Shepard and Metzler's (1971) seminal study, the dependency of latency durations on disparity magnitude result replicated here has been viewed as suggesting that participants have to incre- mentally rotate a memory representation of one shape, comparing it continuously for alignment with the percept of the other shape. This is consistent with the accounts that several of our participants volunteered when informally asked about how they had solved the task.

Although the matching group of participants appeared to perform slightly better than the oddity group of participants, the differences were not significant. However, the men, on average, exhibited a significantly faster mental rotation than did the women, $F(4,84)=7.33, p<.01$. This sex difference has been often found with visual rotation tasks and is thought to be associated with a generally somewhat superior male spatial ability (Pellegrino \& Kail, 1982; Potegal, 1982). Our results, including this detail, thus closely replicate the typical mental rotation effect as described by many other authors (e.g., Corballis, 1988; Shepard \& Cooper, 1982). Indeed, using the same matching-to-sample procedure used here, Förster, Gebhardt, Lindlar, Siemann, and Delius (1995) similarly reproduced this invariance effect with several other mirror-image stimulus pairs.

Arbitrary odd stimuli. The results of the different shapes were not significantly different. Figure 3 thus displays the pooled results. Inspection of the figure shows that the participants made relatively few errors and that they were also fast in choosing. Even at the $0^{\circ}$ comparison rotation the latencies were shorter than with the mirror-image stimuli. Much differently from that task, neither error rates nor

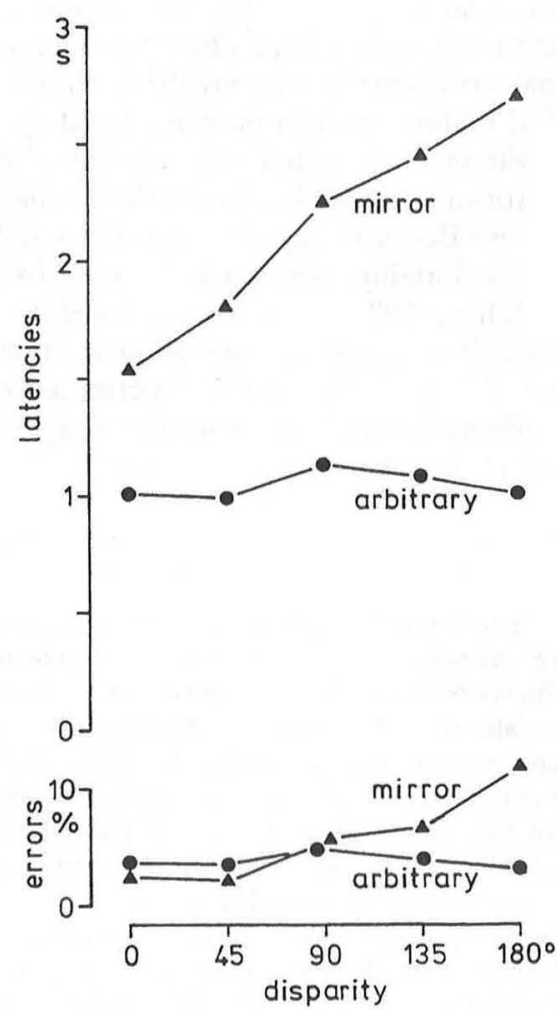

Figure 3. Mirror-image odd shapes and arbitrary odd shapes in Experiment 1 with humans. Mean error rates and choice latencies as a function of orientation disparity. 
reaction times were significantly affected by the orientation disparities of comparison stimuli.

The matching group of participants, however, evinced a slightly but just significantly shorter average choice latency than the oddity group ( $0.82 \mathrm{~s}$ vs. $1.27 \mathrm{~s})$, Wilcoxon MannWhitney test, $W(10,10)=128, p<.05$. This is consistent with a so-called same-faster effect frequently found with humans in experiments requiring same-different decisions about visual stimuli (Farrell, 1985). A small male advantage in average accuracy was not significant.

The absence of a mental rotation effect reproduces the modal result of several previous experiments on rotational invariance involving nonmirror odd stimuli (Corballis \& Nagourney, 1978; Eley, 1983; Tilgner \& Hauske, 1980; White, 1980). With such stimuli, mental rotation effects appear to surface only when sample and odd comparison stimuli are chosen to be closely similar and thus specially difficult to discriminate (Förster et al., 1995).

\section{Experiment 2: Mirror-Image Odd Stimuli in Pigeons}

In this experiment we investigated how pigeons would perform in an orientation invariance task involving mirrorimage shapes. Specifically, would they exhibit a mental rotation effect similar to that shown by human participants in the corresponding part of Experiment 1? To prepare for the orientation invariance tests, we began this experiment with the training on the basic discrimination tasks. This training took the place of the verbal instructions given to human participants. During this acquisition phase, all shapes constituting a given triplet had the same orientation, meaning that no disparity was involved. When the basic matching and oddity task had been mastered by the birds, rotational invariance was tested with stimulus triplets incorporating mirror-image odd shapes and the standard orientation disparities. Because pigeons have been found to be excellent at memorizing many visual stimuli by rote (von Fersen \& Delius, 1989; Vaughan \& Greene, 1984), it seemed advisable to repeat the orientation invariance testing with a shape that the subjects had experienced previously only at $0^{\circ}$ orientation and then with two shapes that were totally novel to the pigeons.

\section{Method}

Training. Ten domestic pigeons (Columba livia) of the homing variety served as subjects. They were all adult animals obtained from local breeders. The sex of 9 animals was determined postmortem at a later date. There were 4 females and 5 males. The subjects were maintained at approximately $85 \%$ of their normal weights throughout the experiments and kept in individual cages in a brightly lit (12:12-hr light/dark schedule) and well-ventilated animal room. Half of the pigeons were randomly assigned to a matching group and half to an oddity group.

The conditioning panel of the previous experiments (see Figure 1) was enclosed with walls and a ceiling and was provided with a semitransparent door. This converted it into $40 \times 40 \times 40 \mathrm{~cm}$ conditioning chamber. The birds were first trained to peck the plainly illuminated keys for grain access with a conventional hand-shaping procedure. Through a judicious temporary disabling of preferred keys, the pigeons learned to peck each and all keys. Mixed grain reward was offered for $3 \mathrm{~s}$ with a solenoid-operated feeder. It rose under the opening of an alcove $(6 \times 5 \times 8 \mathrm{~cm})$ located on the floor beneath the middle key (see Figure 1). The rewards were signaled with additional lighting as described earlier; punishment again consisted of a 3-s period of darkness.

Discrimination training proceeded with 20 nondisparity stimulus triplets assembled from Shapes 1 to 10 and their mirror images as odds, all at their $0^{\circ}$ orientations (see Figures 1 and $2 \mathrm{~A}$ ). Except for some details, the procedure was closely similar to that used with the human subjects in the preceding experiments. Intertrial intervals initially lasted $3 \mathrm{~s}$ but were gradually lengthened to $15 \mathrm{~s}$. To begin with, the subjects needed to peck both the sample key and a comparison key only once for reinforcement to follow. As their performance improved, the observing response demanded on the sample key was gradually increased to 15 pecks.

There were 40 trials per daily session, with each of the 20 stimulus triplets being presented twice. However, during the first 25 sessions, when necessary, poor performances with given stimulus triplets were counteracted on an individual basis by increasing the frequency of the relevant triplets in the succeeding session to the detriment of better learned ones. From the 5th session onward, a correction procedure was used (i.e., after incorrect responses, the presentations of the same stimulus triplet was repeated until the subject produced a correct choice). Training continued until the pigeons consistently yielded more than $80 \%$ correct responses.

The subjects were then further trained for 10 sessions to familiarize them with non- $0^{\circ}$ shape orientations. Eight new triplets based on Shapes 5 and 9 were used for that purpose. Both the sample and comparison shapes of these triplets were congruently rotated by either $45^{\circ}$ or $90^{\circ}$ clockwise. These triplets, not yet involving any disparity (see Figure $2 \mathrm{~B}$ ), were randomly interspersed twice per session among 12 of the normal $0^{\circ}$ triplets used previously. They were those based on Shapes 1, 2, 3, 4, 5, and 9 and were also shown twice per session.

Familiar stimuli rotations. When training was complete, testing for rotational invariance began. Eight triplets again based on Shapes 5 and 9 and their mirror images, but now with the orientation of the comparison shapes no longer coinciding with that of the sample shapes, replaced the previous triplets with tilted shapes. The new triplets, shown again twice per session among the normal triplets, had the sample shape in its normal $0^{\circ}$ orientation, but the comparison shapes rotated by either $45^{\circ}$ and $90^{\circ}$ clockwise. The aforementioned correction procedure was inoperative with these, and all later, test triplets. After 10 sessions eight more test triplets again based on Shapes 5 and 9 but with the comparison forms now rotated by $135^{\circ}$ and $180^{\circ}$ clockwise (see the top of Figure $2 \mathrm{C}$ ) replaced the nondisparity triplets based on Shapes $1,2,3$, and 4 . Thus, all 20 triplets were then based on Shapes 5 and 9, with the five orientation disparities $0^{\circ}, 45^{\circ}, 90^{\circ}, 135^{\circ}$, and $180^{\circ}$ being equally frequent. Each triplet was presented twice per session for 10 sessions.

For the next 10 sessions, we used 10 triplets based on Shape 8 and its mirror image, which the subjects only knew as constituents of normal $0^{\circ}$ disparity training sets. The comparison stimuli were now shown at all five standard orientation disparities. These triplets replaced the aforementioned triplets based on Shape 5, whereas those based on Shape 9 were retained.

Novel stimuli rotations. In another series of 10 sessions, all 20 triplets used were based on Shapes 11 and 12 and their mirror images, all totally new to the subjects, at all five comparison orientations (see the bottom of Figure 2C). Note that Shape 8 used earlier and Shape 11 used here were also used in Experiment 1 with human participants. 


\section{Results and Discussion}

Training. Nine pigeons took $36-53$ sessions $(M=40.4)$ to reach the $80 \%$ correct criterion. One animal was excluded from the experiment because it did not reach criterion within 60 sessions. Apart from this pigeon, the acquisition of the conditional discriminations must be judged as having been comparatively fast (cf. Delius, 1994; Lombardi, Fachinelli, \& Delius, 1984). However, differently from what is usual, the initial procedure was carefully and individually adapted to yield optimal learning. On average, oddity group pigeons needed fewer sessions (36.0 sessions) to reach criterion than matching group pigeons (44.9 sessions), but the difference was not significant, unless the pigeon not reaching criterion was included with a worst rank, Wilcoxon Mann-Whitney test, $W(5,5)=37, p<.05$.

Familiar stimuli rotations. Figure 4 shows the results obtained with Shapes 5 and 9 and their reflections. Pooled averages are shown because there were no significant differences between the shapes. The functions shown were pieced together with the $0^{\circ}, 45^{\circ}$, and $90^{\circ}$ data coming from the first invariance test series and the $135^{\circ}$ and $180^{\circ}$ coming from the second test series (i.e., when the relevant stimulus triplets were first used). Much differently from the human results with mirror-image odd comparison shapes (Experiment 1, Figure 3), the pigeons' error rates and reaction times, despite exhibiting some inverted $V$ trends, were both not significantly affected by the orientation disparities. A small female advantage in performance was not significant. The accuracy superiority of the oddity group over the matching group was relatively larger but also did not reach significance.

The absence of a mental rotation effect in these results could be attributable to the difference in experience that the

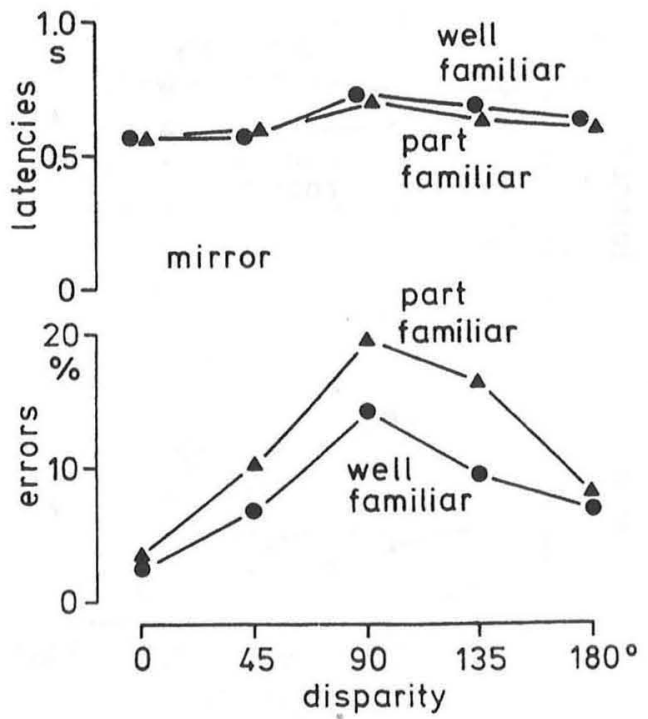

Figure 4. Mirror-image odd stimuli in Experiment 2 with pigeons. Results for highly familiar shapes and, separately, results for a partly familiar shape. Mean error rates and choice latencies are shown as a function of orientation disparity. pigeons and humans had with the relevant stimulus shapes and more specifically with the relevant stimulus triplets. Several facts contradict this explanation. One is the comparatively good performance with the novel comparison shape tilts of $135^{\circ}$ and $180^{\circ}$ with which the pigeons had no previous experience. Another is that a separate analysis of the performance during only the first four trials with each of the new orientation disparity triplets similarly failed to reveal any mental rotationlike effect, although the average accuracy was then lower ( $14.6 \%$ errors) than that corresponding to the overall test (see Figure 4).

Human participants, in any case, do not seem capable of overcoming the mental rotation effect even with prolonged exposure to the task, although initially their performance tends to improve somewhat (Bethell-Fox \& Shepard, 1988; Koriat \& Norman, 1985). This was also, incidentally, our experience; despite much forced commerce with the various stimulus triplets, we continued to have distinct difficulties when classifying the slides involving the larger orientation disparities.

Figure 4 also shows the results relating to Shape 8 , previously known to the birds only in the $0^{\circ}$ orientation. Both the accuracy and latency functions were highly similar to those obtained in the preceding experiment. ANOVAs again revealed no significant disparity dependencies. The recurrence of inverted $V$ trends combined with low error rates on the familiar $0^{\circ}$ disparity triplets was nevertheless striking. Indeed, when the error rates obtained with Stimuli 5,9 , and 8 were pooled, an ANOVA revealed that they were significantly affected by the orientation disparities, but only through a quadratic trend, $F(1,40)=4.55, p<.05$. This is interesting because similar convex parabolic trends occasionally arise in human orientation invariance experiments, sometimes even superimposed on linear mental rotation trends (Jolicoeur, 1985; Förster et al., 1995; Tilgner \& Hauske, 1980; White, 1980). In fact, the results of our Experiment 1 (see Figure 3) were also affected by faint but not significant trends of this kind. However, there does not seem to be any plausible explanation as to what causes them. Moreover, as we show later, they were not a regular feature of pigeon orientation invariance results either.

Novel stimuli rotations. Figure 5 displays the pooled results obtained with the totally novel Shapes 11 and 12; again, there was no significant difference between the results pertaining to the two stimuli. Neither the reaction times nor the error rates were significantly affected by orientation disparity. By not revealing any trace of a mental rotation effect, the results are patently different from those produced by humans with mirror-image stimuli but highly similar to those produced by this latter species with arbitrary, nonmirror stimuli (Experiment 1, Figure 3).

However, because each novel triplet was presented 20 times to each pigeon over the 10 sessions rather than just 4 times over 2 sessions (as with the human participants in Experiment 1), it could still be argued that the pigeons learned to cope with the individual triplets by rote (i.e., without really requiring any invariance competence). This possibility was excluded by examining the performance immediately at the beginning of the tests. The trials at which 


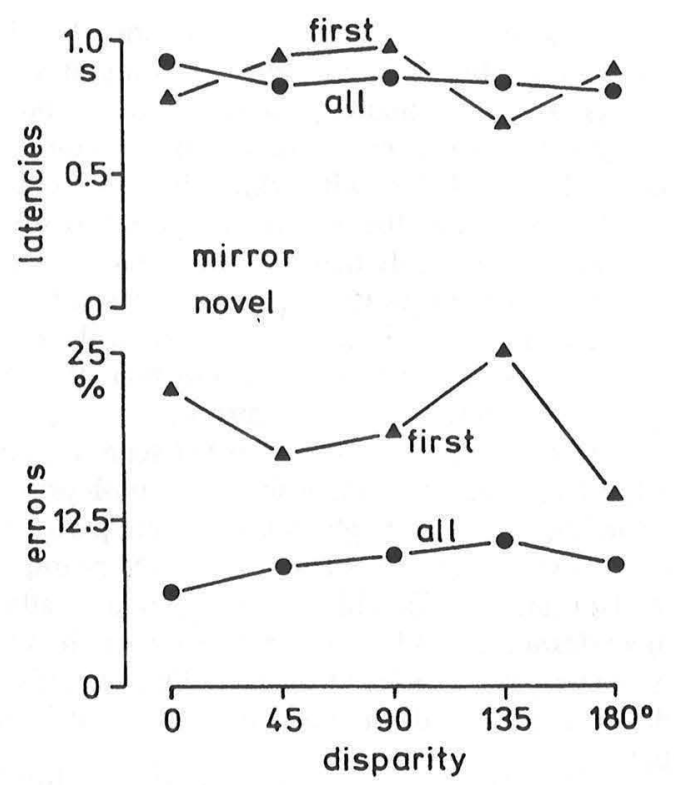

Figure 5. Mirror-image odd stimuli in Experiment 2 with pigeons. Results show novel shapes. Overall mean error rates and choice latencies are shown as a function of orientation disparity. Also shown are mean error rates and median reaction times for first stimulus presentations.

each of the novel stimulus triplets were presented for the first time (i.e., where subjects chose before having ever received any specific reinforcement feedback on them) were singled out. The average accuracy and latency functions calculated on these data and separately displayed in Figure 5 still did not reveal any mental rotationlike effect, even though the error rates were about twice as high during these initial trials than for the overall test. The variance associated with these functions was naturally higher than for the overall functions, as each data point was based only on 36 trials rather than on 720 trials. In fact, the median, instead of the mean, latencies were used in this instance because the latter were too affected by occasional outlier reaction times.

By the end of the second test session, the pigeons had experienced the triplets based on Shapes 11 and 12 four times. Their experience with these novel stimuli was then precisely the same as that of the humans with their mirrorimage stimulus triplets in Experiment 1. The pigeons' corresponding data still did not reveal any mental rotationlike trends, although the mean error level had then diminished to $17.4 \%$.

\section{Experiment 3: Samples Rotated and Arbitrary Stimuli in Pigeons}

In the previous experiment, the sample stimuli were presented with a constant orientation, and the sample shapes were displayed with varying tilts. In the first part of this experiment, we varied the tilt of the sample shapes while presenting the comparison shapes at the standard $0^{\circ}$ orientation. We hoped that this procedural modification would force the pigeons to adopt a different solution strategy that might involve a mental rotationlike processing.

The second part of the experiment was concerned with the response pattern that the pigeons would show when confronted with arbitrary, non-mirror-image shape discriminations. Recall that with this kind of stimuli, humans do not normally evince a mental rotation effect (Experiment 1, Figure 3). Would pigeons possibly also differ from humans when performing this particular task?

\section{Method}

Samples rotated. The subjects, apparatus, and procedure were as before. The subjects completed a series of 10 sessions with 20 stimulus triplets based on Shapes 3 and 4 and their mirror images served. Shape 3 was known to the pigeons only in its $0^{\circ}$ orientation. The sample shape in these triplets was now presented rotated by either $0^{\circ}, 45^{\circ}, 90^{\circ}, 135^{\circ}$, or $180^{\circ}$. The identical and mirrorimage comparison patterns were now conversely always displayed in the standard $0^{\circ}$ orientation (see Figure 2E).

Arbitrary odd stimuli. The stimulus triplets used for this part of the experiment incorporated Shapes 1 and 2 as sample and matching comparison stimuli. Either Shapes 3, 4, 5, 6, or 10 served as odd, arbitrary comparisons, with each shape appearing equally often. The comparison shapes occurred at the five tilt disparities (see Figure 2D). The stimulus triplets were the same as those used with human participants in Experiment 1, arbitrary stimuli. Each triplet was presented twice per session, and there were 10 sessions.

\section{Results and Discussion}

Samples rotated. The results relating to the two sample Shapes 3 and 5 were statistically indistinguishable. As can be seen in Figure 6, the mean accuracy and speed of the pigeons with this modified orientation disparity task was closely similar to that obtained in Experiment 2 with novel
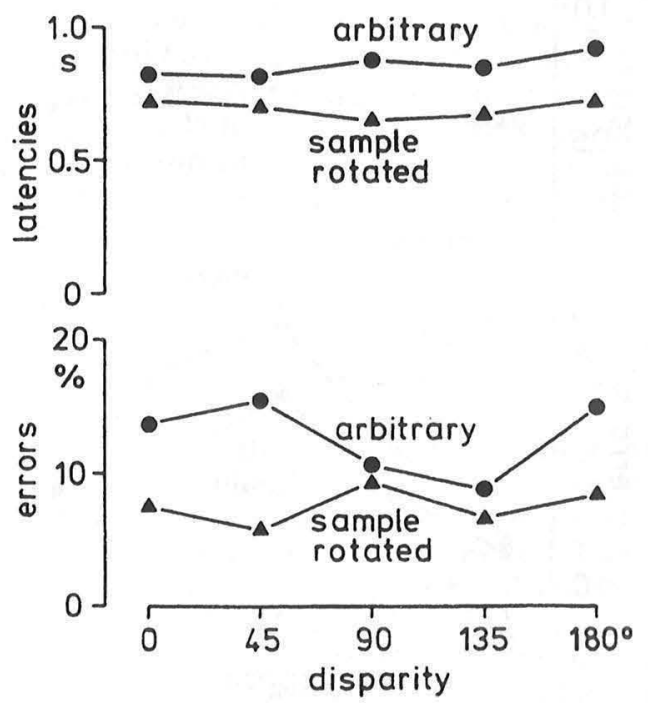

Figure 6. Mirror-image odd shapes, sample rotated, in Experiment 3 with pigeons. Separately, arbitrary odd comparison shapes are shown. Mean error rates and choice latencies are shown as a function of orientation disparity. 
stimuli (see Figure 5). Although not separately shown, this also applied specifically to the first trial performance. It was remarkable that the pigeons' orientation invariance performance was not importantly affected by the fact that the sample shapes, instead of the comparison shapes, were now rotated to achieve the usual orientation disparities.

Although no parallel experiment was formally run with humans, we experienced that the rotated sample task incorporated a disparity-dependent rise in discrimination difficulty resembling that observed in the rotated comparison shapes task used in Experiment 1, which yielded a strong mental rotation effect (see Figure 3, mirror-image shapes).

Arbitrary odd stimuli. The outcome of this part of the experiment is also shown in Figure 6. As with all the pigeon results thus far, neither the error rates nor the response latencies varied significantly with the rotational disparities of the comparison shapes. Except for a slightly but not significantly higher overall error rate, the orientation disparity functions obtained were comparable to those of Experiment 2 (see Figures 4 and 5) and those obtained in Experiment 1 with human participants and using the same arbitrarily different shapes (see Figure 3 ). The ease with which the pigeons switched from discriminating mirrored shapes to discriminating arbitrary shapes and back to discriminating mirrored shapes in the next experiment is noteworthy. In the current experiment, they in fact produced an overall average error rate of only $15.7 \%$ during the first presentations of the arbitrary shape triplets. This score compares well with the initial accuracy achieved with novel shapes in Experiment 2 (see Figure 5).

Overall, the oddity group subjects were again just significantly better in accuracy than the matching group (10.5\% and $17.6 \%$ errors), Wilcoxon Mann-Whitney test, W(4, $5)=28, p<.05$. This recurring trend coincides with one frequently reported in the pigeon literature (Carter \& Werner, 1978; Delius 1994). It is the opposite of that found with humans in Experiment 1, a point to which we return later. Sex differences in performance were again minimal and in fact contrary to those observed in the previous experiments.

Therefore, it does not seem to make any appreciable difference to the performance of pigeons whether reflected or arbitrary stimuli are used as comparison stimuli. Regardless of the stimulus material used, pigeons show an orientation invariance performance consistently devoid of any mental rotation effect.

\section{Experiment 4: Delayed Comparisons and Mirrored Samples in Pigeons}

Several of the experiments that have demonstrated mental rotation effects in humans, although not all and actually not ours (Experiment 1), have used successive procedures that required the subjects to compare the engrams of past sample stimuli with present percepts of comparison stimuli (Roldan \& Phillips, 1980; Shepard \& Cooper, 1982; White, 1980). Would pigeons perhaps evince a mental rotation effect if they were forced to refer to shape memories at the discrim- ination stage? Before the appropriate tests could be run, the birds had to be trained to cope with delays between sample and comparison presentations.

The other procedure used in this experiment also confronted the pigeons with a difficult task in an attempt to force them to reveal a mental rotation effect. The design of the task was influenced by the observation that in the previous experiments, mirror images of the shapes illustrated in Figure 1 occurred only as odd comparison stimuli (compare Figure 2). We now used a more balanced design wherein mirror images also served as sample and identical shapes. Standard shapes in turn also served as odd shapes.

\section{Method}

Delayed comparisons. The subjects and apparatus were the same as those used before. The triplets were based on Shapes 11 and 12 and had already been used in Experiment 2 (see the bottom of Figure 2C). However, now the sample shapes were turned off as soon as the subjects had completed the observing response requirements and the comparison stimuli came on (0-s delay). This was so for 10 sessions. There were then 5 sessions with 1 -s delay, 5 sessions with 2-s delay, and 10 sessions with 5-s delay. At the end of this delay training, all 9 pigeons performed at levels above the $80 \%$ correct criterion. A first test series of 10 sessions used triplets based on Shapes 8,9 , and their reflections. The comparison shapes were presented with a 5-s delay and at all the usual five orientation disparities. These shapes were well known to the pigeons in all orientations from the previous experiments. Because of that, 20 analogous triplets based on Shapes 13, 14 (see Figure 1), and their mirror images, all of which were new to the pigeons, were used in an additional series of 10 sessions.

Mirrored samples. Because the pigeons had performed poorly in the preceding test, they were retrained for 10 sessions with the triplets assembled from Shapes 13 and 14 last used in that test but using the no-delay procedure of the earlier experiments. For the test series of 10 sessions that followed, these stimulus triplets were replaced by others assembled from Shapes 4 and 6 and their mirror images. These shapes occurred alternatively in their standard form or in their reflected form. In turn, the respective shapes occurred as matching identical and odd reflected comparison shapes, both rotated $0^{\circ}, 90^{\circ}$, or $180^{\circ}$ with respect to the samples (see Figure $2 \mathrm{~F}$ ). Each combination occurred in an odd-right and an odd-left combination, resulting in 24 different triplets. These were shown twice in each of the sessions consisting of 48 trials.

\section{Results and Discussion}

Delayed comparisons. The results are presented in Figure 7. Although faint rising trends were noticeable in both the accuracy and speed functions belonging to Shapes 8 and 9 and a more marked inverted $V$ trend was apparent in the accuracy results belonging to novel Shapes 13 and 14, these trends were not statistically significant. In this instance there was a significant difference between the overall mean error rates pertaining to the two familiar stimuli, Shape 9 triplets leading to less errors than Shape 8 triplets $(11.3 \%$ and $25.4 \%$ ), Wilcoxon's test, $T(9)=38, p<.05$, perhaps because the pigeons had more previous experience with the former than the latter shape. Because the difference concerned only the level of accuracy, the data are depicted 


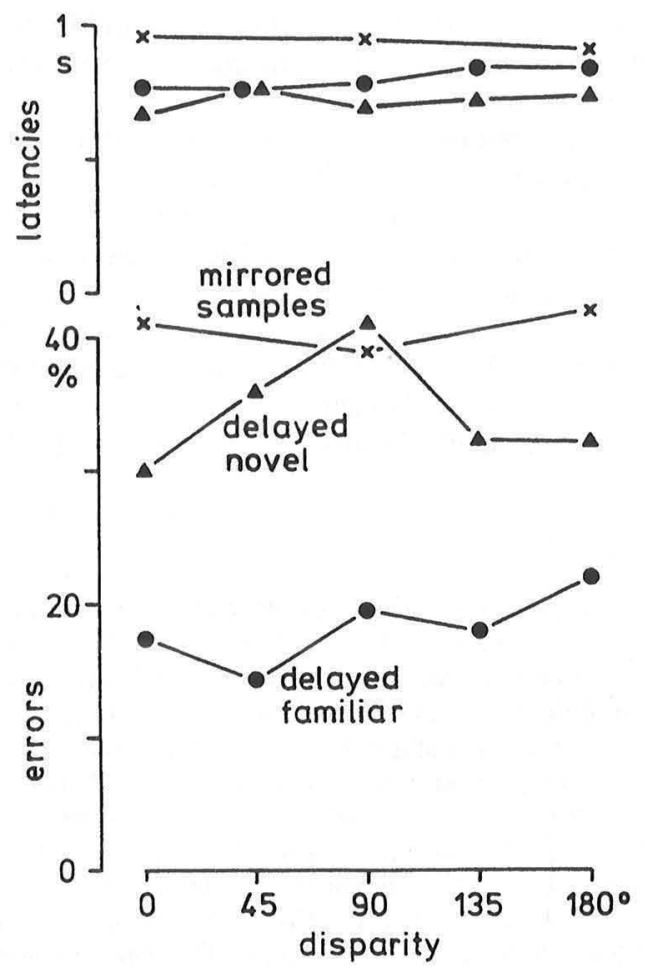

Figure 7. Mirror-image odd stimuli, comparison shapes presented with 5-s delay (familiar shapes and novel shapes), in Experiment 4 with pigeons. Separately, mirrored sample shapes are shown. Mean error rates and choice latencies are shown as a function of orientation disparity.

pooled in Figure 7. There were no significant differences between the results corresponding to novel Shapes 13 and 14. However, during this test, the oddity group of subjects again made significantly fewer errors than did the matching group (32.1\% vs. $40.0 \%$ ), Wilcoxon Mann-Whitney test, $W(4,5)=30, p<.01$. We return to this topic later.

Compared with the novel shape results of Experiment 2 (see Figure 5), the overall error rates were higher here, Wilcoxon's test, $T(9)=43, p>.01$. This is consistent with the general finding that delays between sample and comparison presentations result in a deterioration of choice accuracy in pigeons (short-term memory decay; Grant, 1981). The latencies, however, were not significantly different. In any case, a procedure modification demanding reliance on short-term memorization of the sample shape failed to yield a mental rotation effect in pigeons, even though it obviously involved some increase in task difficulty (see also Delius \& Hollard, 1987).

Mirrored samples. The retraining yielded a rapid reattainment of the $80 \%$ accuracy criterion by all but 1 nonmatching subject, which became ill and had to be excluded. However, during the test the accuracy was generally poor, and all but one of the remaining 8 pigeons did not attain the $80 \%$ criterion again. The overall average error rate was $41.4 \%$ (see Figure 7). Nevertheless, neither error rates nor reaction times varied significantly with angular disparity. The reaction times were slightly but not significantly longer than in the previous experiments. The error rates connected with triplets presenting the sample shapes in the more familiar nonreflected version (i.e., as shown in Figure 1) were significantly lower than those connected with the triplets incorporating the less familiar mirror-image sample shapes ( $46.8 \%$ and $37.5 \%$ errors), Wilcoxon's test, $T(8)=$ $35, p<.01$.

The fact that both the original shapes and their mirror images occurred as sample and also as comparisons clearly made the task difficult for the pigeons: The error rates were only slightly less than chance $(50 \%)$. The experimenters also found the task demanding; the double use of mirror images was an obviously confusing factor. It was nevertheless unmistakable that for them the difficulty rose with increasing orientation disparities. The pigeons, however, did not show any trace of a mental rotation effect.

Although perhaps only suggesting that pigeons, like humans, find mirror images confusing, the poor, almostchance level performance during this experiment serves a purpose. It thoroughly refutes the odd possibility that unintended artifacts might have played a role in the pigeons' surprisingly good orientation invariance recorded in the previous experiments because the methods used were identical.

\section{Experiment 5: Mirror-Image Discriminability in Pigeons and Humans}

The comparison between humans and pigeons up to this point can be summarized by stating that both species show a similar orientation invariance behavior except when mirror-image shapes are involved. In orientation disparity tasks requiring the latter discrimination, humans show a mental rotation effect and pigeons definitely do not. The peculiar difficulty that the former have with discriminating mirror images even when no orientation disparities are involved, a well-documented phenomenon, is arguably an important antecedent for the occurrence of the effect (Corballis, 1988; Förster et al., 1995). Might the effect's absence in pigeons be due to the fact that they, notwithstanding the results of the preceding experiment, find mirror images comparatively easier to discriminate than do humans? Some circumstantial evidence suggests that pigeons probably can discriminate mirror-image patterns well (Corballis \& Beale, 1976; Vaughan \& Greene, 1984), but other researchers (Lohmann et al., 1988; Todrin \& Blough, 1983) have found that pigeons still have some difficulties with discriminating mirror-image shapes. However, none of these studies involved adequately direct comparisons with humans. The experiment we now report was intended to redress this deficit.

\section{Method}

Pigeons. All 9 pigeons used in the earlier experiments participated; the pigeon that had been ill at the end of the last experiment had recovered and was used in this experiment. The same conditioning apparatus and procedures as before were used. Because the terminal performance during the previous experiment had been poor, the subjects were retrained for 10 sessions using stimulus $0^{\circ}$ 
disparity triplets based on Shapes 3 and 4, well known to the pigeons. A test series lasting for 10 sessions was then run using 40 stimulus triplets assembled from the shapes illustrated in Figure 8. There were five basic shapes (middle), five similar shapes (right), and five reflected shapes (left). All shapes had the same surface area, were new to the pigeons, and were always shown in the $0^{\circ}$ orientation (i.e., without any orientation disparities). The sample was always a basic shape; the matching comparison was the identical shape. The nonmatching comparison for one kind of combination was the corresponding reflected shape; for the other kind of combination it was the corresponding similar shape. Each stimulus combination occurred once as an odd-right and once as an odd-left triplet.

Humans. The participants were 20 university students (10 women and 10 men, mean age $=24$ years). Half of them were assigned to a matching-to-sample group; the other half were assigned to an oddity-from-sample group. The apparatus and procedure were the same as those used in Experiment 1, except that the stimulus triplets used were precisely those used with the pigeons. Two sessions of 40 trials each were run consecutively, separated by only a brief pause.

\section{Results and Discussion}

Pigeons. Figure 8 compares the performance between the mirror image and the arbitrary pattern discrimination task. On average, there was a slight tendency for pigeons to make more errors and to be slower with discriminating reflected odd-shaped triplets than with similar odd-shaped triplets, but these differences were not statistically significant. The lack of any significant difference between the mirror and arbitrary condition supports the view that for pigeons, the reflected shapes were nearly as easy to discriminate as the similar shapes. This conclusion was tempered by the fact that the subjects, until the present experiment, had had extensive experience with mirror-image discriminations and only a passing one with discriminating arbitrary shapes, namely, during Experiment 3. However, in that experiment, the pigeons had little difficulty in switching

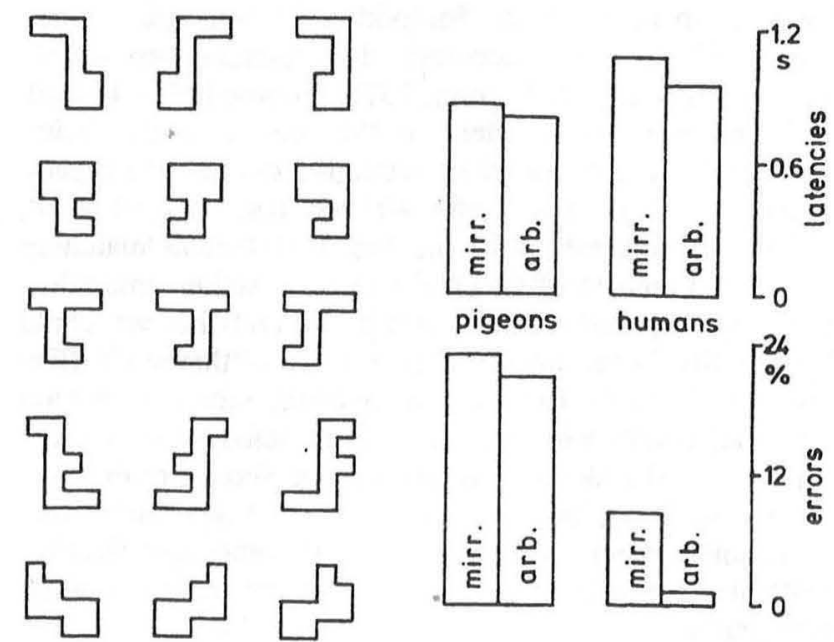

Figure 8. Mirror (mirr.) and arbitrary (arb.) shape discrimination in Experiment 5 with pigeons and humans. Left, stimuli used. Right, comparison of mean error rates and reaction times. from one type of discrimination to the other, suggesting that the differential experience may not have mattered that much here either.

Humans. Figure 8 compares the performance between the mirror image and the arbitrary pattern discrimination task. The somewhat higher average error rate with the mirror-image discrimination was just significant, Wilcoxon's test, $Z(20)=1.7, p<.05$, but the difference in average latencies was not. Otherwise, in accordance with the same-faster effect already mentioned in conjunction with Experiment 1, the mean latencies of the matching group participants were significantly shorter than those of the oddity group participants $(0.81 \mathrm{~s}$ and $1.30 \mathrm{~s}$, respectively), Wilcoxon Mann-Whitney test, $W(10,10)=145, p<$ .01 .

The obvious conclusion that humans have comparatively greater difficulties than pigeons in discriminating mirror shapes rather than arbitrary shapes is somewhat constrained by the absolute differences in performance obtained. The pigeons began with an overall mean accuracy barely above chance level (45.3\% errors) during the first 2 sessions and took 10 sessions to average the last 2 sessions with $11.1 \%$ choice errors. The human participants, having the benefit of verbal instructions and better learning abilities, averaged only $9.8 \%$ errors during their only 2 sessions. What effect such absolute differences in performance have on the relative discrimination performances is hard to evaluate. However, the earlier experiments also produced findings congruent with the present ones. Humans had poorer performance (latencies) when discriminating mirror shapes than when discriminating arbitrary shapes at $0^{\circ}$ disparity (see Figure 1), Wilcoxon Mann-Whitney test, $Z(22,20)=$ $1.98, p<.05$ (compare also Förster et al., 1995), whereas pigeons produced closely similar performances with both of these stimulus types (compare Figures 5 and 6). Thus, until perhaps more exact experiments definitely prove otherwise, it is reasonable to assume that the absence of a mental rotation effect in pigeons might be because they find mirror images, relative to arbitrary shapes, easier to discriminate than do humans.

\section{General Discussion}

Although we could easily reproduce the typical mental rotation effect with humans using a task involving the discrimination of misaligned mirror-image shapes (Experiment 1, Figure 3), we were unable to obtain such an effect with pigeons using a nearly identical task (Experiment 2, Figures 4 and 5). This was the case even when the data sets analyzed were selected so that both pigeons and humans had equal experience with the stimulus shapes used in the respective orientation invariance tests. Throughout, pigeons behaved like humans while discriminating misaligned arbitrary shapes, where these also do not as a rule show a mental rotation effect (Experiment 1, Figure 3). Regardless of various procedural modifications (Experiments 3 and 4), some of which demonstrably made the general task more difficult for them, the pigeons consistently failed to evince 
any mental rotation effect (see Figures 6 and 7). The additional results are thus in line with the conclusions reached in our first report on the matter (Hollard \& Delius, 1982) and with the results of later experiments done in conjunction with a neurological study (Delius \& Hollard, 1987). They also are consistent with the independent orientation invariance findings reported by Lombardi (1986; see also von Fersen \& Delius, 1989). The performance of pigeons in matching-to-sample and oddity-from-sample discrimination is not affected by orientation discrepancies between sample and comparison stimuli, even when mirror-image shapes serve as odd stimuli.

The pigeon orientation invariance results we have reported have been occasionally questioned because they relied on the command of a generalized matching-to-sample or oddity-from-sample strategy by these animals. Until recently, it was often thought that pigeons were actually incapable of mastering such conceptlike rules (Herrnstein, 1985; Mackintosh, 1983). This belief was based on the results of certain earlier, and in retrospect, inadequately conceived experiments. A number of more recent experiments, besides the current ones, have established beyond doubt that pigeons are capable of learning relational matching and oddity rules (Lombardi et al., 1984; Macphail \& Reilly, 1989; Wright, Cook, Rivera, Sands, \& Delius, 1988).

It has also been suggested that because pigeons exhibit response decrements when tested with shapes at orientations different from those to which they were conditioned, or that because pigeons can learn to distinguish different orientations of a shape, our conclusions could not be correct (Cerella, 1990). As already explained elsewhere (Lombardi $\&$ Delius, 1990), the objection is based on a misinterpretation. Our claim that pigeons do not show mental rotation in situations in which humans do does not include the assumption that they are invariably insensitive to orientation. Indeed, we also have shown that pigeons can learn to discriminate differently oriented identical shapes (Delius \& Emmerton, 1978; Lohmann et al., 1988). When humans discriminate misaligned arbitrary shapes, they, like pigeons, do not show a mental rotation effect (Experiment 2). This, however, does not mean that they do not perceive the orientation differences involved; it only means that they are not obstructed by them when solving the task at hand. It seems conceivable that pigeons behave likewise, except that they in some way manage to extend the strategy to mirrorimage shapes, something that humans apparently are not able to do.

In our experiments, pigeons yielded relatively constant and short latencies while producing relatively high and variable error rates. Humans, acquiescing to the instructions about accurate responding, yielded comparatively low and constant error rates while producing comparatively long and variable latencies. This difference in performance characteristics has repeatedly induced the suggestion that if humans were coaxed to behave more like pigeons in this respect, they might also fail to evince a mental rotation effect. Motivated by this argument, T. Schultz and M. Remy (personal communication, 1983) conducted an experiment similar to our Experiment 1 using mirror-image odd stimuli. Their participants, unlike ours, were given stern instructions to respond as fast as possible. The mean latencies they obtained were accordingly markedly shorter than in our experiment, but they still revealed a significant but shallower mental rotation effect. However, through an obvious speed-accuracy trade-off, the mean error rates were comparatively large and affected by a steep, highly significant mental rotation effect. The species difference puzzling us is therefore unlikely to be attributable to the use of alternative performance strategies.

The human participants viewed the stimulus array from $50 \mathrm{~cm}$ away; the pigeons, as it is their nature (Wohlschläger, Jäger, \& Delius, 1993), viewed the array from less than 10 $\mathrm{cm}$ away. Thus, humans could easily encompass the display components with minor ocular saccades. Pigeons, as documented by videographic recordings, scanned the stimuli on the keys with ample head movements. Thus, although humans could rely on a virtually instantaneous image acquisition, pigeons were forced to use a sequential image completion. Could these different intake modes be responsible for the species difference in orientation invariance? Probably not, as ample evidence indicates that a successive stimulus presentation in humans does not prevent the occurrence of a mental rotation effect (Roldan \& Phillips, 1980; Shepard \& Cooper, 1982; White, 1980). Might pigeons, unlike humans, have compensated the varying shape orientations with congruent head tilts? The aforementioned videographic records showed that, although the pigeons varied their head orientation when viewing the individual stimuli, they only rarely did so by more than $30^{\circ}$ from the upright. Furthermore, these tilts bore no relation to the orientation of the shapes. Also, varying head tilts in humans has been found to have only minor effects on mental rotation performance (Corballis, Nagourney, Shetzer, \& Stefanatos, 1978).

The fact that pigeons and humans appear to differ in how they cope with the matching and oddity tasks instrumentalized here has also been forwarded as an obscure explanation for the species-dependent invariance results. There is actually a recurring tendency for oddity-from-sample trained pigeons to be more accurate than matching-to-sample trained ones (Carter \& Werner, 1978; Delius, 1994). Indeed, this trend was also evident in the present study, being comfortably significant when averaging over all the pigeon experiments, Wilcoxon Mann-Whitney test, $W(4,5)=30$, $p>.01$. It contrasts with the fact that human matching participants tend to often yield shorter reaction times than oddity participants (Farrell, 1985), a trend that we could also identify in the human experiments of this study (Experiments 1 and 5). However, none of the human or pigeon invariance results were significantly affected by an interaction between the identity-oddity variable and the orientation disparity variable. Therefore, regardless of how interesting this peculiar species difference may be otherwise (Delius, 1994), it is unlikely to be the cause of the mental rotation discrepancy.

The hypothesis that we think still best explains why pigeons are locally more efficient than humans in achieving an orientation invariant performance is that they, unlike 
humans, do not normally need to recur to the serial processing that yields the retarding mental rotation effect. Independent evidence indicates that the factor that causes humans to exceptionally evince a mental rotation effect when discriminating arbitrary shapes is that these must be selected to be particularly difficult to distinguish in the absence of any orientation disparity (Förster et al., 1995). It is thus reasonable to assume that the property determining the fact that mirror-image shapes invariably elicit a mental rotation effect in humans must be that they are relatively difficult to discriminate even when identically aligned (Corballis \& Beale, 1976; compare Corballis, 1988). The fact that pigeons do not recur to serial processing when dealing with mirror-image shapes must mean that they, unlike humans, do not experience any special discrimination difficulties with them (Hollard \& Delius, 1982).

That is precisely what is conveyed by the contrasting human and pigeon results of Experiment 5, despite its limitations. Note that this argument does not require that pigeons must find mirror-image and arbitrary shapes equally difficult to discriminate. It only demands that compared with humans, pigeons must have relatively fewer difficulties with them than with arbitrary shapes. The mere fact that pigeons have been shown to have some difficulties with discriminating mirror images in some contexts (Lohmann et al., 1988; Todrin \& Blough, 1983) is not detrimental because it does not exclude the possibility that humans could have even more pronounced difficulties. Our argument, however, suggests that if one could identify arbitrary shapes pairs that were sufficiently difficult for pigeons to discriminate prior to any orientation disparities, then these stimuli, when presented with such disparities, might force them to show a mental rotation effect. If such an experiment was successful, the easy-to-discriminate mirror-images hypothesis would be indirectly strengthened.

Regardless of how the issue is resolved, the wider question of why humans are less efficient than pigeons when discriminating disoriented mirror-image shapes is already inescapable. The most obvious answer would seem to be that the processing of mirror images in the geniculocortical visual system predominating in mammals differs in some relevant way from that in the tectoectostriatal visual system preeminent in birds (Delius \& Hollard, 1987; Emmerton, 1983; compare Remy \& Güntürkün, 1991). Recently, however, it has been shown that baboon monkeys reveal a humanlike mental rotation effect when discriminating mirror-image shapes they see in their right optical hemifield (left cortex processing) and a pigeonlike flat invariance function when they see the shapes in their left optical hemifield (right cortex processing; Vauclair, Fagot, \& Hopkins, 1993). A far less extreme hemispheric differentiation regarding visual orientation invariance performance may indeed be present in humans (Corballis \& Sargent, 1989; Dittuno \& Mann, 1990). The monkey results show that at least in principle, the mammalian, specifically the primate, geniculocortical system is just as suited for parallel processing of mirror-image orientation invariance as is the avian tectoectostriatal system.

Attempts to relate the orientation invariance abilities to global neuroanatomical structures may thus not be heuristically fruitful. Perhaps a better approach is to first focus on the question of how any ensemble of neurons can cope with such tasks. Goebel (1990) devised an artificial neuronal network model that achieves a mental rotationlike performance using interneurons that force incremental steps of orientational change on the pattern information that is being recognized by the network. Remarkably, Georgopoulos, Lurito, Petrides, Schwartz, and Massey (1989) described a mental rotationlike activity of motor-cortical neuron ensembles in macaque monkeys that could well be operating on the basis of such a mechanism. A parallelization of the serially operating twitch units that would free the performance of Goebel's model from the mental rotation effect seems theoretically possible, but the fact is that this has not yet been actually implemented. Such modeling could well provide more incisive insights into the pigeon-human difference occupying us here.

Meanwhile, we can nevertheless speculate why humans do not benefit from the potential capability of the primate visual system demonstrated by the above-cited baboon results. Humans' deficit may have to do with bioevolutionary adaptations. Hollard and Delius (1982) argued that the excellence of orientation invariance in pigeons may have arisen phylogenetically because of the special demands that a typical avian lifestyle makes on them. It generally requires them to operate visually on the horizontal ground plane both in flight and walking. On this plane, objects and subjects have no prescribed orientation. The visual systems of birds must thus have been under selective pressure to achieve efficient visual recognition regardless of relative orientations. Our more remote primate ancestors are thought to have been highly arboreal. They would thus have been exposed to some of the same pressures as birds regarding visual orientation invariance competences. Humans, because of their ground-bound, upright stance, mainly operate visually on the vertical plane, where they and most objects have standardized orientations determined by gravity. The lesser selective pressure implied might not have sufficed to ensure the retention of an invariance mechanism that, although efficient in performance, is bound to be neurally elaborate and costly. The hominids thus may have secondarily lost a piece of visuospatial intelligence that obviously still adorns the minds of baboons and pigeons.

\section{References}

Bethell-Fox, C. E., \& Shepard, R. N. (1988). Mental rotation: Effects of stimulus complexity and familiarity. Journal of Experimental Psychology: Human Perception and Performance, 14, 12-23.

Carter, D. E., \& Werner, T.J. (1978). Complex learning and information processing by pigeons: A critical analysis. Journal of the Experimental Analysis of Behavior, 29, 565-601.

Cerella, J. (1990). Shape constancy in the pigeon: The perspective transformations decomposed. In M. L. Commons, R. J. Herrnstein, S. M. Kosslyn, \& D. B. Mumford (Eds.), Quantitative analysis of behavior: Behavioral approaches to pattern recognition and concept formation (pp. 145-163). Hillsdale, NJ: Erlbaum. 
Cooper, L. A. (1975). Mental rotation of random two-dimensional shapes. Cognitive Psychology, 7, 20-43.

Corballis, M. C. (1988). Recognition of disoriented shapes. Psychological Review, 95, 115-123.

Corballis, M. C., \& Beale, I. L. (1976). The psychology of left and right. Hillsdale, NJ: Erlbaum.

Corballis, M. C., \& Nagourney, B. A. (1978). Latency to categorize alphanumeric characters as letters or digits. Canadian Journal of Psychology, 32, 186-188.

Corballis, M. C., Nagourney, B. A., Shetzer, L. I., \& Stefanatos, G. (1978). Mental rotation under head tilt: Factors influencing the location of the subjective reference frame. Perception \& Psychophysics, 24, 263-273.

Corballis, M. C., \& Sargent, J. (1989). Hemispheric specialization for mental rotation. Cortex, 25, 15-25.

Delius, J. D. (1979). [Insensitivity to shape orientation in pigeons]. Unpublished raw data.

Delius, J. D. (1994). Comparative cognition of identity. In P. Bertelson, P. Eelen, \& G. d'Ydevalle (Eds.), International perspectives on psychological science: Ongoing research. 1: Leading themes (pp. 25-40). Hillsdale, NJ: Erlbaum.

Delius, J. D., \& Emmerton, J. (1978). Sensory mechanism related to homing in pigeons. In $\mathrm{K}$. Schmidt-Koenig \& W. T. Keeton (Eds.), Animal migration, navigation and homing (pp. 35-41). Berlin: Springer.

Delius, J. D., \& Hollard, V. D. (1987). Orientation invariance of shape recognition in forebrain-lesioned pigeons. Behavioral Brain Research, 23, 251-259.

Ditunno, P. L., \& Mann, V. A. (1990). Right hemisphere specialization for mental rotation in normal and brain-damaged subjects. Cortex, 26, 177-188.

Eley, M. G. (1983). Symbol complexity and symbol identification with rotated symbols. Acta Psychologica, 53, 27-35.

Emmerton, J. (1983). Vision. In M. Abs (Ed.), Physiology and behavior of the pigeon (pp. 245-266). San Diego, CA: Academic Press.

Farrell, B. (1985). "Same"-“different" judgments: A review of current controversies in perceptual comparison. Psychological Bulletin, 98, 419-456.

Förster, B., Gebhardt, R. P., Lindlar, K., Siemann, M., \& Delius, J. D. (1995). Mental rotation effect: A function of elementary stimulus discriminability? Manuscript submitted for publication.

Gellermann, C. W. (1933). Chance orders of alternating stimuli in visual discrimination experiments. Journal of Genetic Psychology, 42, 206-208.

Georgopoulos, A. P., Lurito, J. T., Petrides, M., Schwartz, A. B., \& Massey, J. T. (1989). Mental rotation of the neuronal population vector. Science, 234, 234-236.

Goebel, R. P. (1990). The mathematics of mental rotations. Journal of Mathematical Psychology, 34, 435-440.

Gonzales, R. C., \& Wintz, P. (1987). Digital image processing (2nd ed.). Reading, MA: Addison-Wesley.

Grant, D. S. (1981). Short term memory in the pigeon. In N. E. Spear \& R. R. Miller (Eds.), Information processing in animals: Memory mechanisms (pp. 227-256). Hillsdale, NJ: Erlbaum.

Herrnstein, R. J. (1985). Riddles of natural categorization. Philosophical Transactions of the Royal Society, Series B, 308, 129 144.

Hollard, V. D., \& Delius, J. D.: (1982). Rotational invariance in visual pattern recognition by pigeons and humans. Science, 218 , 804-806.

Jolicoeur, P. (1985). The time to name disoriented natural objects. Memory \& Cognition, 13, 289-303.
Koriat, A., \& Norman, J. (1985). Mental rotation and visual familiarity. Perception \& Psychophysics, 37, 429-439.

Kosslyn, S. M., Pinker, S., Smith, G. E., \& Schwartz, S. P. (1979). On the demystification of mental imagery. Behavioral and Brain Sciences, 2, 535-581.

Lohmann, A., Delius, J. D., Hollard, V. D., \& Friesel, M. (1988). Discrimination of shape reflections and shape orientations by Columba livia. Journal of Comparative Psychology, 102, 3-13.

Lombardi, C. M. (1986). Shape oddity recognition of pigeons is independent of shape orientation. Revista Mexicana del Analisis Experimental de la Conducta, 5, 265-272.

Lombardi, C. M., \& Delius, J. D. (1990). Size invariance in visual pattern recognition by pigeons. In M. L. Commons, R. J. Herrnstein, S. M. Kosslyn, \& D. B. Mumford (Eds.), Quantitative analysis of behavior: Behavioral approaches to pattern recognition and concept formation (pp. 41-56). Hillsdale, NJ: Erlbaum.

Lombardi, C. M., Fachinelli, C., \& Delius, J. D. (1984). Oddity of visual patterns conceptualized by pigeons. Animal Learning \& Behavior, 12, 2-6.

Mackintosh, N. J. (1983). Conditioning and associative learning. Oxford, England: Clarendon Press.

Macphail, E. M., \& Reilly, S. (1989). Rapid acquisition of a novelty versus familiarity concept by pigeons (Columba livia). Journal of Experimental Psychology: Animal Behavior Processes, 15, 242-252.

Messner, R., \& Szu, H. (1985). An image processing architecture for real time generation of scale and rotation invariant patterns. Computer Vision, Graphics and Image Processing, 31, 50-66.

Pellegrino, J. W., \& Kail, R. (1982). Process analyses of spatial aptitude. In R. J. Sternberg (Ed.), The psychology of human intelligence (pp. 311-365). Hillsdale, NJ: Erlbaum.

Petrusic, W. M., Varro, L., \& Jamieson, D. G. (1978). Mental rotation validation of two spatial ability tests. Psychological Research, 40, 139-148.

Potegal, M. (Ed.). (1982). Spatial abilities, developmental and physiological foundations. San Diego, CA: Academic Press.

Pylyshyn, Z.W. (1981). The imagery debate: Analogue media versus tacit knowledge. Psychological Review, 88, 16-45.

Remy, M., \& Güntürkün, O. (1991). Retinal afferents to the tectum opticum and the $\mathrm{n}$. otpicus principalis thalami in the pigeon Journal of Comparative Neurology, 305, 57-70.

Roldan, C. E., \& Phillips, W. A. (1980). Functional differences between upright and rotated images. Quarterly Journal of Experimental Psychology, 32, 397-412.

Shepard, R. N., \& Cooper, L. A. (1982). Mental images and their transformations. Cambridge, MA: MIT Press.

Shepard, R. N., \& Metzler, J. (1971). Mental rotation of threedimensional objects. Science, 171, 701-703.

Tilgner, R. D., \& Hauske, G. (1980). Untersuchung zur Rotationsinvarianz bei der Erkennung von Buchstaben [Investigations into the rotation invariance during the recognition of letters]. Zeitschrift für angewandte und experimentelle Psychologie, 27, $147-162$

Todrin, D. C., \& Blough, D.S. (1983). The discrimination of mirror-image forms by pigeons. Perception \& Psychophysics, 34, 397-402.

Vauclair, J., Fagot, J., \& Hopkins, W. D. (1993). Rotation of mental images in baboons when the visual input is directed to the left cerebral hemisphere. Psychological Science, 4, 99-103.

Vaughan, W., \& Greene, S. L. (1984). Pigeon visual memory capacity. Journal of Experimental Psychology: Animal Behavior Processes, 10, 256-271. 
von Fersen, L., \& Delius, J. D. (1989). Long-term retention of many visual patterns by pigeons. Ethology, 82, 141-155.

White, M. J. (1980). Naming and categorization of tilted alphanumeric characters do not require mental rotation. Bulletin of the Psychonomic Society, 15, 153-156.

Wohlschläger, A., Jäger, R., \& Delius, J. D. (1993). Head and eye movements in unrestrained pigeons (Columba livia). Journal of Comparative Psychology, 107, 313-319.
Wright, A. A., Cook, R. G., Rivera, J. J., Sands, S. F., \& Delius, J. D. (1988). Concept learning by pigeons: Matching-to-sample with trial unique video picture stimuli. Animal Learning \& Behavior, 16, 436-444. 